Pacific Journal of Mathematics 


\title{
ON INFINITE GROUPS
}

\author{
W. R. ScotT
}

1. Introduction. Several disconnected theorems on infinite groups will be given in this paper. In $\S 2$, a generalization of Poincare's theorem on the index of the intersection of two subgroups is proved. Other theorems on indices are given. In $\$ 3$, the theorem [3, Lemma 1 and Corollary 1] that the layer of elements of infinite order in a group $G$ has order 0 or $o(G)$ is generalized to the case where the order is taken with respect to a subgroup. In $\$ 4$, it is shown that the subgroup $K$ of an infinite group $G$ as defined in [3] is overcharacteristic [2]. In $\$ 5$, characterizations are obtained for those Abelian groups $G$, all of whose subgroups $H$ (factor groups $G / H$ ) of order equal to $o(G)$ are isomorphic to $G$ (in this connection, compare with [7]). Again the Abelian groups, all of whose order preserving endomorphisms are onto, are found (see [6]).

2. Index theorems. If $H$ is a subgroup of $G$, let $i(H)$ denote the index of $H$ in $G$. The cardinal of a set $S$ will be denoted by $o(S)$.

THEOREM 1. Let $H_{\alpha}$ be a subgroup of $G, \alpha \in S$. Then

$$
i\left(\cap H_{\alpha}\right) \leq \prod_{i}\left(H_{\alpha}\right)
$$

Proof.

$$
g_{1} g_{2}^{-1} \in \cap H_{\alpha}
$$

if and only if

$$
g_{1} g_{2}^{-1} \in H_{\alpha} \text { for all } \alpha \in S \text {. }
$$

Thus each coset of $\cap H_{\alpha}$ is the intersection of a collection of sets consisting of one coset of $H_{\alpha}$ for each $\alpha$, and the conclusion follows.

COR OllaRY 1. (Poincaré) The intersection of a finite number of subgroups of finite index is again of finite index.

Received December 23, 1953.

Pacific J. Math. 5 (1955), 589-598 
Corollary 2. If $i(H)=B$, then $G$ has a normal subgroup $K$ such that $i(H) \leq B^{B}$.

Proof. Let $N(H)$ denote the normalizer of $H$, and $C l(H)$ the conjugate class of $H$. Then

$$
H \subseteq N(H), \quad o(C l(H))=i(N(H)) \leq B
$$

Thus if $K$ is the intersection of the conjugates of $H$, Theorem 1 gives $i(K) \leq B^{B}$.

REMARKS. For every infinite cardinal $A$, there is a simple group $G$ of order $A$ (for example, the "alternating" group on $A$ symbols). Thus $G$ has no subgroups of index less than or equal to $B$ if $2^{B}<A$. In particular, if $A$ is such that $B<A$ implies $2^{B}<A$, then $G$ has no subgroup of index less than its order $A$. This is in sharp contrast to the behaviour of Abelian groups, which have $2^{A}$ subgroups of index $B$ for $\boldsymbol{x}_{0} \leq B \leq A, A>\boldsymbol{x}_{0}$ [4]. It is an unsolved problem as to whether there exists a group $G$ of order $A$ with no subgroups of order $A$, for $A>\mathbf{x}_{0}$.

Let $U$ denote the point set union, and + and $\sum$ direct sums (the lattice union of subgroups will not be used). If $T$ is a nonempty subset of a group $G$, let

$$
i_{R}(T)=\min o(S) \text { such that } U T x_{\alpha}=G, \alpha \in S
$$

Define $i_{L}(T)$ similarly, and let $i(T)$ be the smaller of $i_{R}(T)$ and $i_{L}(T)$.

THEOREM 2. If $H_{i}, i=1, \cdots, n$, are subgroups of $G$ such that $i\left(H_{i}\right) \geq$ $A \geq \boldsymbol{\aleph}_{0}$, then $i\left(\mathrm{UH}_{i}\right) \geq A$.

Proof. The theorem is true for $n=1$. Induction on $n$. If, contrary to the theorem, $i\left(\cup H_{i}\right)<A$, then, say,

$$
G=\bigcup_{a \in S}\left(\bigcup_{i=1}^{n} H_{i}\right) x_{\alpha}
$$

with $o(S)<A$. Since $i\left(H_{1}\right) \geq A$, there exists an $x \in G$ such that

$$
H_{1} x \cap\left(U_{\alpha} H_{1} x_{\alpha}\right)
$$

is empty. Hence

$$
H_{1} x \subseteq \mathrm{U}_{a}\left(\mathrm{U}_{2}^{n} H_{i}\right) x_{a}
$$


Therefore

$$
\bigcup_{i=1}^{n} H_{i} \subseteq \bigcup_{i=2}^{n} H_{i}\left(e \cup\left(\bigcup_{\alpha} x_{\alpha} x^{-1}\right)\right)=\bigcup_{\beta \in S^{\prime}}\left(\bigcup_{i=2}^{n} H_{i}\right) x_{\beta},
$$

where $o\left(S^{\prime}\right)<A$. Hence

$$
G=\bigcup_{a \in S}\left(\bigcup_{i=1}^{n} H_{i}\right) x_{\alpha}=\bigcup_{\alpha \in S} \underset{\beta \in S^{\prime}}{\bigcup} \bigcup_{i=2}^{n} H_{i} x_{\beta} x_{\alpha}=\bigcup_{\gamma \in S} \bigcup_{i=2}^{n} H_{i} x_{\gamma}, o\left(S^{\prime \prime}\right)<A
$$

This contradicts the induction hypothesis. Hence the theorem is true.

REMARK. For every infinite cardinal $A$, there is a group $G$ of order $A$, containing an increasing sequence $\left\{H_{n}\right\}$ of subgroups, each of index $A$, such that $\cup H_{n}=G$.

$$
\text { Let } 1 / A=0 \text { for } A \geq \boldsymbol{x}_{0} \text {. }
$$

THEOREM 3. If $H_{i}$ is a proper subgroup of $G,(i=1, \cdots, n)$ and $\sum 1 / i\left(H_{i}\right) \leq$ 1 , then $\cup H_{i} \neq G$.

Proof. Let $H_{1}, \cdots, H_{r}$ have finite index, the others infinite index (if $r=0$, the theorem follows immediately from Theorem 2). Let

$$
D=\bigcap_{1}^{r} H_{i}
$$

Then $U$ has finite index in $G$, and it is well known that $\left(\cup_{i}^{r} H_{i}\right) \cap D x$ is empty for some $x \in G$. Hence, if $\cup_{1}^{n} H_{i}=G$, then $D x \subseteq \cup_{r+1}^{n} H_{i}$, whence $\cup_{r+1}^{n} H_{i}$ has finite "index" in contradiction to Theorem 2. Therefore $\cup_{1}^{n} H_{i} \neq G$.

3. Layers. Let $T$ be a subset of $G$, and let $n$ be a positive integer. Let

$$
\begin{aligned}
& L(n, T)=\left\{g \mid g^{n} \in T, g^{r} \notin T \text { for } 0<r<n\right\}, \\
& L(\infty, T)=\left\{g \mid g^{n} \notin T, n=1,2, \cdots\right\} .
\end{aligned}
$$

For $T=e$, the $L(n, T)$ have been called layers. The following theorem generalizes [3, Lemma 1].

THEOREM 4. Let $G$ be an infinite group, $H$ a subgroup, $P$ a set of primes and 


$$
S=\left(\bigcup_{p \in P} \underset{\lambda}{\cup} L(\lambda p, H)\right) \cup L(\infty, H)
$$

Then $o(S)=0$ or $o(G)$.

Proof. Deny the theorem. Let $x \in S$. If $x \in L(\lambda p, H)$ then $x^{\lambda} \in L(p, H)$. Hence we may assume that $x \in L(\infty, H)$ or $x \in L(p, H), p \in P$.

Case 1. $o(N(x))=o(G)$, where $N(x)$ is the normalizer of $x$. Then $o(N(x)-$ $S)=o(G)$. If $y \in N(x)-S$, then $y^{r} \in H$ for some $r$ such that $(r, p)=1$ (if $p$ exists). If $x y \notin S$ then also $(x y)^{n} \in H$ for some $n$ such that $(n, p)=1$ (if $p$ exists ). Thus

$$
(x y)^{r n}=x^{r n} y^{r n} \in H,
$$

and $x^{r n} \in H$. But $(r n, p)=1$ if $p$ exists, and, in any case, we have a contradiction. Hence $x y \in S$ and

$$
o(S) \geq o(x(N(x)-S))=o(N(x)-S)=o(G)
$$

a contradiction.

Case 2. $o(N(x))<o(G)$. Then $o(C l(x))=o(G)$.

Case 2.1. $o(H)=o(G)$. Then $o(G)$ right cosets of $N(x)$ intersect $H$. Thus there are $o(G)$ elements of the form $h^{-1} x h$. But if $\left(h^{-1} x h\right)^{n} \in H$ then $x^{n} \in H$, whence $n=\lambda p$ and $h^{-1} x h \in S$. Therefore $o(S)=o(G)$, a contradiction.

Case 2.2. $o(H)<o(G)$. We have, since $o(S)<o(G)$,

$$
o(G)=o(C l(x))=\sum_{\substack{(n, p)=1 \\ n<\infty}} o(C l(x) \cap L(n, H)) .
$$

If $o(G)=\mathbf{x}_{0}$, and $o(x)=\infty$, then since $H$ is finite,

$$
C l(x) \subseteq L(\infty, H) \subseteq S,
$$

a contradiction. If $o(G)=\boldsymbol{x}_{0}$, and $o(x)=m$, then $C l(x) \cap L(n, H)$ is empty for $n>m$. Hence, by (1), there exists, regardless of the size of $o(G)$, an $n$ such that $(n, p)=1$ and

$$
o(C l(x) \cap L(n, H))>o(H) o(S) .
$$




$$
A(n, T)=\left\{g \mid g^{n} \in T\right\}
$$

Then $A(n, H) \supseteq L(n, H)$, hence

$$
o(C l(x) \cap A(n, H))=\sum_{h} o(C l(x) \cap A(n, h))>o(H)_{o}(S) .
$$

Hence there exists an $h_{0} \in H$ such that

$$
o\left(C l(x) \cap A\left(n, h_{0}\right)>o(S) .\right.
$$

There is then a $b \in G$ such that $\left(b^{-1} x b\right)^{n}=h_{0}$, whence

$$
x \in C l(x) \cap A\left(n, b h_{0} b^{-1}\right)
$$

If

$$
q \in C l(x) \cap A\left(n, b h_{0} b^{-1}\right)
$$

then

$$
q^{n}=b h_{0} b^{-1}=x^{n}
$$

Hence if $q^{r} \in H$, then

$$
x^{n r}=q^{n r} \in H
$$

and $p \mid n r$, whence $p \mid r$. Thus $q \in S$ in any case. We have

$$
\begin{aligned}
o(S) \geq o\left(C l(x) \cap A\left(n, b h_{0} b^{-1}\right)\right) & =o\left(b\left(C l(x) \cap A\left(n, h_{0}\right)\right) b^{-1}\right) \\
& =o\left(C l(x) \cap A\left(n, h_{0}\right)\right)>o(S) .
\end{aligned}
$$

This contradiction shows that the theorem is true.

COROLLARY. If $H$ is a subgroup of the group $G$, then $o(L(\infty, H))=o(G)$ or 0 .

Proof. In Theorem 4 , let $P$ be the empty set.

4. An over-characteristic subgroup. Neumann and Neumann [2] have defined a subgroup $K$ of $G$ to be over-characteristic in $G$ if and only if (i) $K$ is normal, and (ii) $G / K \cong G / H$ implies $K \subseteq H$. 
Define (see [3]) a subgroup $K$ of an infinite group $G$ as follows. Let $E(x)$ be the set of $g \in G$ such that $x$ is not in the subgroup generated by $g$, and let $K$ be the set of $x \in G$ such that $o(E(x))<o(G)$.

Тнеовем 5. If $G$ is infinite, and $K$ is defined as above, then $K$ is an overcharacteristic subgroup of $G$.

Proof. (i) $K$ is normal since it is fully characteristic [3, Theorem 6].

(ii) Let $G / K \cong G / H$.

Case 1. $K$ is finite. Then [ 3 , Corollary 3 to Theorem 8 ]

$$
K_{2}=K(G / K)=e .
$$

Ilence $K(G / H)=e$. Now

$$
o(G / H)=o(G / K)=o(G) .
$$

If there exists a $k \in K-H$, then

$$
o(E(k H)) \leq o(E(k))<o(G)=o(G / H) .
$$

Hence $k H \in K(G / H)$. This is a contradiction. Hence $K \subseteq h$, and $K$ is overcharacteristic.

Case 2. $K$ is infinite. Then [3, Theorem 5] $K$ is a $p^{\infty}$ group, and [3, Theorem 8] $G / K$ is finite. If there exists a $k \in K-H$ then

$$
k^{\prime p^{n}}=k
$$

implies $k^{\prime} \in K-H$, and

$$
o\left(k^{\prime} H\right) \geq p^{n+1} \text {. }
$$

This contradicts the finiteness of $G / H$. Therefore $K \subseteq H$, and since $G / K$ is finite, $K=H$. Hence $K$ is over-characteristic.

5. Abelian groups with special properties. ${ }^{1}$ If $G$ is an Abelian group such that $0 \subset H \subset G$ implies $G \cong H$ for subgroups $H$, then it is trivial that $G$ is 0 or cyclic of prime or infinite order, and conversely. This naturally leads to the problem of finding those groups which possess the following property:

1 For the facts used without proof in this section, see [1]. 
$\left(P_{1}\right) G$ is Abelian, and if $H$ is a subgroup of $G$ such that $o(H)=o(G)$ then $G \cong H$.

THEOREM 6. $G$ has property $\left(P_{1}\right)$ if and only if $(\mathrm{i}) G$ is finite Abelian, (ii) $G$ is a $p^{\infty}$ group, (iii) $G$ is a direct sum of cyclic groups of order $p, p a$ fixed prime, (iv) $G$ is infinite cyclic, or (v) $G$ is the direct sum of a nondenumerable number of infinite cyclic groups.

Proof. If $G$ is of one of the above five types, then it is either trivial or well-known that $G$ has property $\left(P_{1}\right)$.

Conversely, suppose that $G$ is infinite and has property $\left(P_{1}\right)$. Let $T$ be the torsion subgroup of $G$.

Case 1. $o(T)<o(G)$. Then (see, for example, [3, proof of Theorem 9, Case 1]) there is a free Abelian subgroup $H$ of $G$ such that $o(H)=o(G)$. Hence $G \cong H$. If the rank of $G$ is non-denumerable, we are done. If the rank of $G$ is countable, then $G$ is countable and contains an infinite cyclic subgroup. By $\left(P_{1}\right), G$ is infinite cyclic.

Case 2. $o(T)=o(G)$. Then $G \cong T$, that is, $G$ is periodic. If $G_{p}$ is a nonzero $p$-component of $G$, then $G=G_{p}+H_{p}$, hence $G \cong G_{p}$ or $G \cong H_{p}$, a contradiction unless $H_{p}=0$. Hence $G$ is a p-group. Thus $G=D+R$, where $D$ is a divisible (that is, $n D=D$ ) and $R$ a reduced (no divisible non-zero subgroups) p-group. Hence $G \cong R$ or $G \cong D$, that is $G$ is reduced or divisible.

Case 2.1. $G$ is a divisible p-group. Then $G=\sum C_{a}$ where $C_{a}$ is a $p^{\infty}$ group. If there is more than one summand, then there is a subgroup

$$
H=C^{*}+\sum C_{a},
$$

$\alpha \neq \alpha_{0}$, where $C^{*}$ is a proper subgroup of $C_{a_{0}}$. Hence $o(H)=o(G)$, but $H$ is not divisible, a contradiction. Therefore $G$ is a $p^{\infty}$ group in this case.

Case 2.2. $G$ is a reduced p-group. Then $G$ has a cyclic direct summand $C$ of order, say, $p^{n}$. Zorn's lemma may be applied to sets $S$ of cyclic groups $C_{a}$ of order $p^{n}$ such that $\sum C_{a}, C_{\alpha} \in S$, exists and is pure in $G$ (that is, a servant subgroup of $G$ ). There is then a maximal such set $S^{*}$, and if $K=\sum C_{a}$, $C_{a} \in S^{*}$, then $K$ is a pure subgroup of bounded order. Hence $K$ is a direct summand, $G=K+A$. It is clear that $A$ has no cyclic direct summands of order $p^{n}$. This implies, by property $\left(P_{1}\right)$, that $o(A)<o(G)$, hence $G \cong K$. If, now, $n>1$, there is a subgroup $H$ of $K$ of order $o(G)$ such that $H \nsubseteq K$. Therefore $n=1$. 
Theorem 6 has a dual.

$\left(P_{2}\right) G$ is Abelian, and $o(G / H)=o(G)$ implies $G \cong G / H$.

THEOREM 7. $G$ has property $\left(P_{2}\right)$ if and only if $(\mathrm{i}) G$ is finite Abelian, (ii) $G$ is infinite cyclic, (iii) $G$ is a direct sum of cyclic groups of order $p$, (iv) $G$ is a $p^{\infty}$ group, or $(\mathrm{v}) G$ is the direct sum of a non-denumerable number of $p^{\infty}$ groups.

Proof. If $G$ is of one of the above five types, then it is clear that $G$ has property $\left(P_{2}\right)$.

Conversely suppose that $G$ is infinite and has property $\left(P_{2}\right)$.

Case 1. $o(G / T)=o(G)$. Then, by $\left(P_{2}\right) G$ is torsion-free. Let $C$ be a cyclic subgroup of $G$. Then $2 C$ is cyclic, and $G / 2 C$ has an element of order 2 , hence $o(G / 2 C)<o(G)$. Therefore $o(G)=\boldsymbol{\aleph}_{0}$, and $o(G / C)$ is finite, hence $G$ is cyclic.

Case 2. $o(G / T)<o(G)$. Hence $o(T)=o(G)$. Let $S$ be a maximal linearly independent set of elements, $B$ the subgroup generated by $S$ ( set $B=0$ if $S$ is empty). Then $T$ ก $B=0$, hence $T$ is isomorphic to a subgroup of $G / B$, and therefore $o(G / B)=o(G)$. But $G / B$ is periodic, hence $G$ is periodic. It follows, just as in the proof of Theorem 6 , that $G$ is either a divisible or a reduced p-group.

Case 2.1. $G$ is a divisible p-group. Then $G=\sum C_{a}$, where $C_{a}$ is a $p^{\infty}$ group. If the number of summands is non-denumerable, we are done. If not, then $G$ is homomorphic to a $p^{\infty}$ group, and $o(G)=\boldsymbol{\aleph}_{0}$. Therefore by $\left(P_{2}\right), G$ is a $p^{\infty}$ group.

Case 2.2. $G$ is a reduced p-group. Then, almost exactly as in Case 2.2 of Theorem 6, it follows that $G$ is the direct sum of cyclic groups of order $p$.

REMARK. Szélpál [7] has shown that if $G$ is an Abelian group which is isomorphic to all proper quotient groups, then $G$ is a cyclic group of order $p$ or a $p^{\infty}$ group. Theorem 7 may be considered as a generalization of this theorem.

Szele and Szélpál [6] have shown that if $G$ is an Abelian group such that every non-zero endomorphism is onto, then $G$ is a cyclic group of order $p$, a $p^{\infty}$ group, or the rationals. The following theorem may be considered as a generalization. 
$\left(P_{3}\right) G$ is Abelian, and if $\sigma$ is an endomorphism of $G$ such that $o(G \sigma)=o(G)$ then $G \sigma=G$.

THEOREM 8. $G$ has property $\left(P_{3}\right)$ if and only if $(\mathrm{i}) G$ is finite Abelian, (ii) $G$ is a $p^{\infty}$ group, or (iii) $G$ is the group of rationals.

Proof. If $G$ is of one of the above three types, then it is clear that $\left(P_{3}\right)$ is satisfied.

Conversely, suppose that $G$ is an infinite group satisfying $\left(P_{3}\right)$.

Case 1. $G$ is torsion-free. Then if $p G \neq G$ for some $p$, the transformation $g \sigma=p g$ is an isomorphism of $G$ into itself, so that $o(G \sigma)=o(G), G \sigma \neq G$, a contradiction. Hence $p G=G$ for all $p$, and therefore $G=\sum R_{a}$, where $R_{a}$ is is isomorphic to the group of rationals. If there is more than one summand, then there is a projection $\sigma$ of $G$ onto $\sum R_{\alpha}, \alpha \neq \alpha_{0}$, a contradiction. Hence $G$ is the group of rationals.

Case 2. $G$ is not torsion-free. Then $G=A+B$ where $A$ is finite (and nonzero ) or a $p^{\infty}$ group. Thus the projection $\sigma$ of $G$ onto the larger of $A$ and $B$ yields a contradiction unless $B=0$. But in this case, since $G$ is infinite, $G=A$ is a $p^{\infty}$ group.

Finally (compare with Szele [5]) consider the following property.

$\left(P_{4}\right) \quad G$ is Abelian, and if $\sigma$ is an endomorphism of $G$ such that $o(G \sigma)=o(G)$ then $\sigma$ is an automorphism of $G$

Corollary. $G$ has property $\left(P_{4}\right)$ if and only if $(\mathrm{i}) G$ is finite Abelian, or (ii) $G$ is the group of rationals.

\section{REFERENCES}

1. I. Kaplansky, Infinite Abelian groups, Michigan University Publications in Mathematics no. 2, Ann Arbor, 1954.

2. B. H. Neumann and Hanna Neumann, Zwei Klassen charakterischer Untergruppen und ihre Faktorgruppen, Math. Nachr. 4 (1950), $106-125$.

3. W. R. Scott, Groups and cardinal numbers, Amer. J. Math. 74 (1952), 187-197.

4. The number of subgroups of given index in non-denumerable Abelian groups, Proc. Amer. Math. Soc., 5 (1954), 19-22.

5. T. Szele, Die Abelschen Gruppen ohne eigentliche Endomorphismen, Acta. Univ. Szeged. Sect. Sci. Math. 13 (1949), 54-56.

6. T. Szele and I. Szélpál, Uber drei wichtige Gruppen, Acta. Univ. Szeged. Sect. Sci. Math. 13 (1950), $192-194$. 
7. I. Szélpál, Die Abelschen Gruppen ohne eigentliche Homomorphismen, Acta. Univ. Szeged. Sect. Sci. Math. 13 (1949), $51-53$.

UNIVERSITY OF KANSAS 


\section{PACIFIC JOURNAL OF MATHEMATICS}

\section{EDITORS}

\section{H.L. ROY DEN}

Stanford University

Stanford, California

E. HEWITT

University of Washington

Seattle 5, Washington

\section{R. P. DILWORTH}

California Institute of Technology

Pasadena 4, California

* Alfred Horn

University of California

Los Angeles 24, California

\section{ASSOCIATE EDITORS}

\begin{abstract}
H. BUSEMANN
HERBERT FEDERER

MARSHALL HALL
\end{abstract}

\section{P.R. HALMOS}

HEINZ HOPF

ALFRED HORN
R.D. JAMES

BØRGE JESSEN

PAUL LÉVY
GEORGE PÓLYA

J.J. STOKER

KOSAKU YOSIDA
UNIVERSITY OF BRITISH COLUMBIA CALIFORNIA INSTITUTE OF TECHNOLOGY UNIVERSITY OF CALIFORNIA, BER KELEY UNIVERSITY OF CALIFORNIA, DAVIS UNIVERSITY OF CALIFORNIA, LOS ANGELES UNIVERSITY OF CALIFORNIA, SANTA BARBARA MONTANA STATE UNIVERSITY

UNIVERSITY OF NEVADA

OREGON STATE COLLEGE

UNIVERSITY OF OREGON
UNIVERSITY OF SOUTHERN CALIFORNIA STANFORD UNIVERSITY UNIVERSITY OF UTAH WASHINGTON STATE COLLEGE UNIVERSITY OF WASHINGTON

AMERICAN MATHEMATICAL SOCIETY HUGHES AIRCRAFT COMPANY SHELL DEVELOPMENT COMPANY

Mathematical papers intended for publication in the Pacific Journal of Mathematics should be typewritten (double spaced), and the author should keep a complete copy. Manuscripts may be sent to any of the editors. Manuscripts intended for the outgoing editors should be sent to their successors. All other communications to the editors should be addressed to the managing editor, Alfred Horn, at the University of California Los Angeles 24, California.

50 reprints of each article are furnished free of charge; additional copies may be obtained at cost in multiples of 50 .

The Pacific Journal of Mathematics is published quarterly, in March, June, September, and December. The price per volume (4 numbers) is $\$ 12.00$; single issues, $\$ 3.50$; back numbers (Volumes $1,2,3$ ) are available at $\$ 2.50$ per copy. Special price to individual faculty members of supporting institutions and to individual members of the American Mathematical Society: $\$ 4.00$ per volume; single issues, $\$ 1.25$.

Subscriptions, orders for back numbers, and changes of address should be sent to the publishers, University of California Press, Berkeley 4, California.

Printed at Ann Arbor, Michigan. Entered as second class matter at the Post Office, Berkeley, California.

* During the absence of E.G. Straus.

UNIVERSITY OF CALIFORNIA PRESS - BERKELEY AND LOS ANGELES 


\section{Pacific Journal of Mathematics}

\section{Vol. 5, No. $4 \quad$ December, 1955}

Richard Horace Battin, Note on the "Evaluation of an integral occurring in servomechanism theory" ............................. 481

Frank Herbert Brownell, III, An extension of Weyl's asymptotic law for eigenvalues................................. 483

Wilbur Eugene Deskins, On the homomorphisms of an algebra onto Frobenius algebras .................................. 501

James Michael Gardner Fell, The measure ring for a cube of arbitrary dimension ....................................... 513

Harley M. Flanders, The norm function of an algebraic field extension. II ............................................ 519

Dieter Gaier, On the change of index for summable series ............ 529

Marshall Hall and Lowell J. Paige, Complete mappings of finite groups . . . . 541

Moses Richardson, Relativization and extension of solutions of irreflexive relations..................................... 551

Peter Scherk, An inequality for sets of integers .................. 585

W. R. Scott, On infinite groups ........................... 589

A. Seidenberg, On homogeneous linear differential equations with arbitrary constant coefficients ......................................... 599

Victor Lenard Shapiro, Cantor-type uniqueness of multiple trigonometric integrals...

Leonard Tornheim, Minimal basis and inessential discriminant divisors for a cubic field...

Helmut Wielandt, On eigenvalues of sums of normal matrices ... 